\section{Chromatin Threads from Cell Nuclei}

MucH work has been done during the last few years by Mirsky and Ris ${ }^{1,2}$ and by Claude and Potter ${ }^{3}$ on thread-like bodies isolated from cell nuclei, such as those of carp erythrocytes and calf thymus. These bodies are sometimes called chromatin threads ${ }^{3}$ and sometimes, as by Mirsky and Ris, more boldly and specifically "isolated chromosomes". In this Laboratory we have repeated so far as possible from the published data the work described by these authors. We have not been concerned in the first instance with biochemical studies ${ }^{4}$ of these threads, but have tried to find if they can properly be regarded as chromosomes. The purpose of this note is to summarize the work leading to our conclusion that the threads are not isolated chromosomes, but are complex fragments of drawn-out nuclei.

Four methods for disintegrating nuclei have been used : (i) the method of Claude and Potter, namely, grinding with sand; (ii) the method of Mirsky and Ris, in which a suspension of the material is placed in a Waring mixer, where it is subjected to the shearing action of a small propeller-like blade rotating at speeds up to 15,000 r.p.m. ; (iii) suspensions were subjected to shearing action in the narrow annulus between two glass cylinders of nearly equal diameters; (iv) suspensions were treated with ultrasonics. Calf thymus tissue was treated by all four methods and chick erythrocytes by method (ii). The products of treatment were examined in the phasecontrast microscope, in the electron microscope, and in the ordinary mieroscope after fixation and staining. Acetic acid, acetic alcohol, osmium tetroxide and Maximow's mixture (Zenker-formol-osmic) were used as fixatives. For examination in the light microscope, the material was stained with one of the following: acetic-orcein, Ehrlich's hæmatoxylin, or Feulgen counter-stained with naphthol green-light-green. The effects of ultrasonics need be mentioned only briefly as they have no bearing on the main problem; the nuclei were torn into fragments of various shapes, and Feulgen-stained material became somewhat diffused in the process.

By examining samples taken at short intervals during treatment, the other three methods were all found to draw out the nuclei into thread-like formations (Figs. I and 2), which later broke off as isolated threads. Ultimately the suspension consisted entirely of such threads. Further evidence has been obtained that the threads are fragments of drawn-out nuclei. In the intact nuclei of both the thymocytes (lympho-

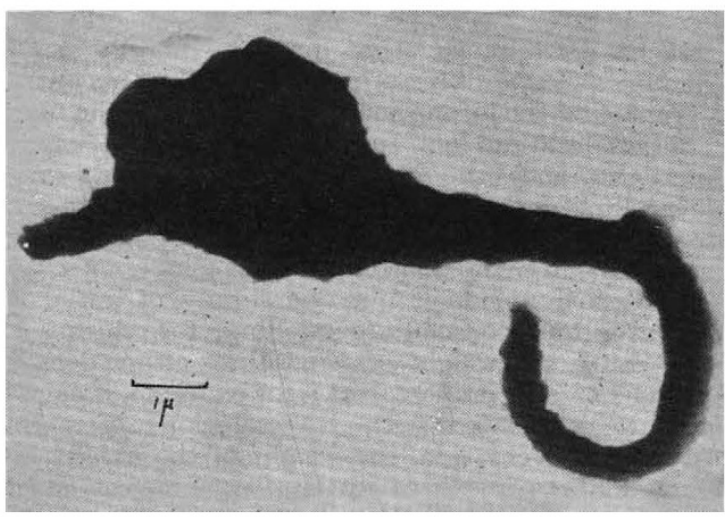

Fig. 1. Electron micrograph of calf thymus nucleus after treatment in the Waring mixer

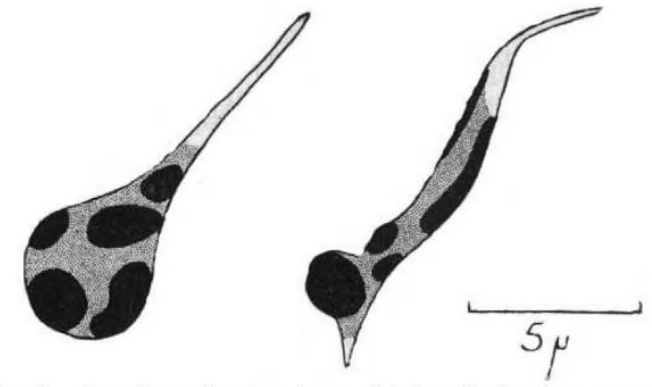

Fig. 2. Drawings showing type of internal structure seen in nuclei and protrusions

cytes) and the larger cells (reticulum cells) of the thymus a certain amount of internal structure can be seen, and it has been noticed that during the earlier stages of treatment these internal structures proceed into the thread-like protrusions of the nuclei (Fig. 2). Mirsky and Ris ${ }^{4}$ have argued that as these threads are of various lengths and sometimes appear to be double, they are isolated metaphase chromosomes. However, the length depends on the method of production and the degree of severity of the treatment; while the apparent double nature of some of them is not confirmed by examination in the electron microscope, which shows the threads to be single.

In considering the possibility of extracting chromosomes from nuclei, it should be remembered that thymocyte nuclei are interphase nuclei from which one would not expect to obtain metaphase chromosomes. I conclude that the threads so far produced by mechanical treatment of nuclei are not chromosomes.

This work will be published in greater detail elsewhere.

I am indebted to Dr. Honor B. Fell, of the Strangeways Laboratory, Cambridge, and to Prof. J. T. Randall, for helpful discussion and advice.

\section{W. G. P. Lamb}

Wheatstone Physics Laboratory, and

Medical Research Council Biophysics Unit, King's College, London, W.C.2.

${ }^{1}$ Mirsky, A. F., and Pollister, A. W., "Frontiers in Cytochemistry", Biological Symposium, 10, 247 (Lancaster, 1943).

${ }^{2}$ Mirsky, A. E., and Ris, H., J. Physiol., 31, 1 (1947).

$s$ Claude, A., and Potter, J. S., J. Exp. Med., 7\%, 345 (1943).

4 Mlirsky, A. E., and Ris, H., Nature, 163, 666 (1949).

\section{Pharmacodynamic Action of Acetylcholine}

THE old 'side-chain' theory of Ehrlich placed unwarranted emphasis on pictorial symbols designed to represent molecular entities. In an interesting recent article, $H$. R. Ing ${ }^{1}$ has suggested that a precise fit between "the drug cation and some macromolecular structure in the living cell is required if a high degree of activity is to be observed". A similar preoccupation with 'skeletal' rather than 'dynamic' properties of autonomic drugs will be found in the recent speculations of Pfeiffer $^{2}$, who has suggested that all drugs which possess parasympathetic stimulant action contain a ketone oxygen group adjacent to an ether oxygen linkage with a methyl-substituted nitrogen at a distance of two saturated carbon atoms. 